\title{
Vorwort zur 5. Auflage
}

Bei der vorliegenden 5. Auflage wurden nicht nur zahlreiche Daten aktualisiert, sondern auch einige Abschnitte überarbeitet oder ergänzt. Der Aufbau des Buches wurde aber beibehalten, der Umfang nur wenig erweitert.

Im Kapitel Die chemische Bindung:

- d-Orbitalbeteiligung in der Nichtmetallchemie

- neue Abbildung und Erläuterung zum MO-Diagramm von $\mathrm{H}_{2} \mathrm{O}$

- Hyperkonjugation, nicht-klassische $\pi$-Bindung; am Beispiel von $\mathrm{POX}_{3}$ und $\mathrm{SF}_{6}$ mit Abbildungen zu MO-Diagrammen.

Im Kapitel Die chemische Reaktion:

- bei den Elektrochemischen Stromquellen wurden die wichtigen Brennstoffzellen ausführlicher behandelt. Neu sind eine Abbildung und eine Tabelle. Aufgenommen wurden auch die aktuellen Lithium-Ionen-Akkumulatoren und der $\mathrm{Na}$ $\mathrm{NiCl}_{2}$-Akkumulator.

Die Elemente der Hauptgruppen:

- Kohlenstoff-Nano-Röhren, dazu eine Abbildung sowie endohydrale Fullerenderivate

- im Abschnitt Umweltprobleme wurden die neuesten Daten des Umweltbundesamtes sowie der Report of Working Group of the Intergovernmental Panel of Climate Change (IPCC) im Internet berücksichtigt. (Literaturangaben im Abschn. Umweltprobleme)

- Korrosionsschutz in Stählen durch Phosphatierung.

Beispiele für weitere Ergänzungen:

- neue Zahlenwerte

Strahlenbelastung; Kernkraftwerke und ihre Leistungen; Bindungsenergien; Dissoziationsenergien von Wasserstoffhalogeniden, Interhalogenverbindungen $\mathrm{XY}$, Chalkogenen und zweiatomigen Molekülen; Elektronenaffinitäten; Chloralkalielektrolyse

- physiologische Eigenschaften und biologische Relevanz $\mathrm{N}_{2} \mathrm{O}, \mathrm{NO}, \mathrm{NO}_{3}^{-}, \mathrm{NaNO}_{2}, \mathrm{Se}$

- neue Modifikationen und Verbindungen sowie Verbindungen mit besonderen Eigenschaften

$\mathrm{HArF} ;\left[\mathrm{AuXe}_{4}\right]^{2+} ; \mathrm{Xe}^{2+} \mathrm{Xe}_{2}^{+}$; das Molekül $\mathrm{P}_{6} ; \mathrm{As}\left(\mathrm{CH}_{3}\right)_{3} ; \mathrm{F}_{3}^{-} ; \mathrm{H}_{2} \mathrm{CO}_{3}$; das Klimagas $\mathrm{SF}_{5}-\mathrm{CF}_{3}$; der neue Supraleiter $\mathrm{MgB}_{2}$; das erste $\mathrm{Au}$ (II)-Fluorid $\mathrm{Au}\left(\mathrm{AuF}_{4}\right)_{2} ; \mathrm{CaTaO}_{2} \mathrm{~N}$ und $\mathrm{LaTaON}_{2}$, Ersatzpigmente für die toxischen CdSCdSe-Pigmente; die Radikale $\mathrm{ClO}$ und $\mathrm{ClO}_{4}$ und ihre Bindung

- aktualisiert wurden die Produktionszahlen wichtiger Nichtmetalle und Nichtmetallverbindungen wie $\mathrm{Cl}_{2}, \mathrm{HCl}, \mathrm{S}, \mathrm{NH}_{3}$ etc. sowie der wichtigsten Gebrauchsmetalle, der Edelmetalle und von Uran. 
Für die bereitwillige Hilfe bei der Ermittlung zahlreicher Daten danken wir: Dr. M. Dalheimer, Bundesanstalt für Geowissenschaften und Rohstoffe, Hannover; Dr. H. Meinke, Verband der Chemischen Industrie e.V., Frankfurt; Dr. H. Pfeiffer, Wirtschaftsvereinigung Stahl, Düsseldorf; Dr. H.-J. Ziesing, Deutsches Institut für Wirtschaftsforschung (DIW), Berlin; den zuständigen Mitarbeitern des Umweltbundesamtes, Berlin.

Auch die gute Zusammenarbeit mit den Mitarbeitern des Verlages soll nicht unerwähnt bleiben.

Berlin

Erwin Riedel

Freiburg

Christoph Janiak

Juli 2002

\section{Vorwort zur 1. Auflage}

Grundlage für dieses Lehrbuch waren die Vorlesungen, die ich im Grundstudium für Studenten der Fachrichtungen Chemie und Lebensmittelchemie an der TU Berlin gehalten habe. Bei der Ausarbeitung des Manuskripts habe ich mich um ein ausgewogenes Verhältnis von Theorie und Stoffchemie bemüht. Ausführlicher werden besonders die Nebengruppenelemente behandelt, für die in der Vorlesung meist nur Zeit für einen Metall-Galopp zur Verfügung steht. Im Aufbau und in der Didaktik wurde darauf geachtet, daß trotz des größeren Stoffumfangs das Buch für das Grundstudium geeignet bleibt.

Das Buch ist in fünf Kapitel gegliedert. In den ersten drei Kapiteln-Atombau, Die chemische Bindung und Die chemische Reaktion - sind die wichtigsten theoretischen Grundlagen dargestellt. Die anderen beiden Kapitel - Die Elemente der Hauptgruppen und Die Elemente der Nebengruppen - enthalten hauptsächlich Stoffchemie außerdem einige theoretische Abschnitte wie Magnetochemie und Komplexverbindungen, die besonders für die Übergangselemente von Bedeutung sind.

Verbindungen und Verbindungsklassen sind durch Fettdruck gekennzeichnet. Wichtige Begriffe und Sachverhalte sind durch Farbdruck hervorgehoben. Auch bei den Abbildungen ist versucht worden, die Farbe informativ und nicht plakativ zu verwenden. Durch Lesen allein des Farbteils in Verbindung mit den Abbildungen sollen die wichtigsten Probleme rasch erfaßt werden können, was das Repetieren erleichtert.

Für die kritische Durchsicht des Manuskripts danke ich Herrn Dr. Wolfgang Paterno. Auch allen Mitarbeitern des Verlags sei für ihr Entgegenkommen und die angenehme Zusammenarbeit gedankt. 